\title{
ON A GENERAL CLASS OF RENEWAL RISK PROCESS: ANALYSIS OF THE GERBER-SHIU FUNCTION
}

\author{
SHUANMING LI, ${ }^{*}$ University of Melbourne \\ JOSÉ GARRIDO, ${ }^{* *}$ Concordia University
}

\begin{abstract}
We consider a compound renewal (Sparre Andersen) risk process with interclaim times that have a $K_{n}$ distribution (i.e. the Laplace transform of their density function is a ratio of two polynomials of degree at most $n \in \mathbb{N}$ ). The Laplace transform of the expected discounted penalty function at ruin is derived. This leads to a generalization of the defective renewal equations given by Willmot (1999) and Gerber and Shiu (2005). Finally, explicit results are given for rationally distributed claim severities.
\end{abstract}

Keywords: Sparre Andersen risk process; $K_{n}$ family of distributions; martingale; Laplace transform; generalized Lundberg equation; defective renewal equation

2000 Mathematics Subject Classification: Primary 62P05

Secondary 60K05

\section{Introduction}

Much of the literature on ruin theory is concentrated on the classical risk model, in which claims occur as a Poisson process. Andersen (1957) let claims occur according to a more general renewal process and derived an integral equation for the corresponding ruin probability. Since then, random walks and queueing theory have provided a more general framework, which has led to explicit results in the case where interclaim times or claim severities have distributions related to the Erlang and phase-type, or more general, $K_{n}$ distributions, whose Laplace-Stieltjes transform is the ratio of a polynomial of degree $k<n$ to a polynomial of degree $n$ (see Willmot (1999)).

$\mathrm{Li}$ and Garrido (2004) considered a risk process with interclaim times being independent and identically Erlang $(n)$ distributed, for $n \in \mathbb{N}^{+}=\{1,2, \ldots\}$. It extends the classical risk model and the Erlang(2) model of Dickson (1998b), Dickson and Hipp (1998), (2001), and Cheng and Tang (2003). Gerber and Shiu (2003), (2005) further extended the theory to generalized Erlang $(n)$ interarrival times (i.e. the distribution is the convolution of $n$ exponential distributions, with possibly different parameters).

The evaluation of the Gerber-Shiu expected discounted penalty function, first introduced in Gerber and Shiu (1998), is now one of the main research problems in ruin theory. Cheng and Tang (2003), Dickson (1998b), Dickson and Hipp (1998), (2001), Gerber and Shiu (2003), (2005), Li (2003), Li and Garrido (2004), and Lin (2003) first derived high-order integrodifferential equations for the expected discounted penalty functions. When integrated iteratively, these produce defective renewal equations. Many ruin-related quantities, e.g. explicit asymptotic,

Received 10 May 2004; revision received 21 March 2005.

* Postal address: Centre for Actuarial Studies, University of Melbourne, Victoria 3010, Australia.

** Postal address: Department of Mathematics and Statistics, Concordia University, Montreal, Quebec H4B 1R6,

Canada. Email address: garrido@mathstat.concordia.ca 
two-sided bounds for ruin probabilities, joint and marginal distributions of the three random variables, the time of ruin, surplus before ruin, and deficit at ruin, as well as their moments, are obtained from these renewal equations by appropriately choosing special penalty functions.

In this paper, we consider the expected discounted penalty function for a broad class of Sparre Andersen risk process, in which the distribution of interclaim times is assumed to belong to the $K_{n}$ family of distributions $\left(n \in \mathbb{N}^{+}\right)$. This general class of distributions includes, as special cases, the Erlang and phase-type distributions, as well as mixtures of these.

\section{Model description and notation}

Consider a continuous-time compound renewal risk process

$$
R(t)=c t-\sum_{i=1}^{N(t)} X_{i}, \quad t \geq 0
$$

(known as Sparre Andersen's model in risk theory), where $c \geq 0$ is the continuous aggregate premium income rate per unit time, while the random sum represents aggregate claims. The $X_{i}$ are independent, identically distributed random variables with common distribution function $P$ (such that $P(0)=0$ ) and density $p$. The variable $X_{i}$ represents the $i$ th claim amount. Denote the $k$ th moment of $X$ by $\mu_{k}=\mathrm{E}\left[X^{k}\right]$ and the Laplace transform (LT) of $p$ by $\hat{p}(s)=\int_{0}^{\infty} \mathrm{e}^{-s x} p(x) \mathrm{d} x$. The counting process $\{N(t), t \geq 0\}$ denotes the number of claims up to time $t$ and is defined as $N(t)=\max \left\{n \in \mathbb{N}^{+}: W_{1}+W_{2}+\cdots+W_{n} \leq t\right\}$, where the interclaim times $W_{i}$ are assumed to be independent and identically distributed with common distribution function $K$ (and corresponding density function $k$ ). Denote by $\hat{k}(s)=\int_{0}^{\infty} \mathrm{e}^{-s x} k(x) \mathrm{d} x$ the LT of $k$.

Furthermore, assume that $\left\{W_{i}\right\}_{i \geq 1}$ and $\left\{X_{i}\right\}_{i \geq 1}$ are independent and that $c \mathrm{E}\left[W_{i}\right]>\mathrm{E}\left[X_{i}\right]$, providing a positive safety loading factor.

The corresponding continuous-time Sparre Andersen surplus process is defined as

$$
U(t)=u+c t-\sum_{i=1}^{N(t)} X_{i}, \quad t \geq 0,
$$

where $u \geq 0$ is the initial surplus. This leads to the definition of the ruin time

$$
T= \begin{cases}\inf \{t \geq 0: U(t)<0\} & \text { if such a } t \text { exists } \\ \infty & \text { otherwise }\end{cases}
$$

and the ultimate ruin probability

$$
\Psi(u)=\mathrm{P}\{T<\infty \mid U(0)=u\}, \quad u \geq 0 .
$$

Consider $F_{3}(x, y, t \mid u)=\mathrm{P}\left\{U\left(T^{-}\right) \leq x,|U(T)| \leq y, T \leq t \mid U(0)=u\right\}$, for $x, y, t \geq 0$, and its corresponding joint probability density function, $f_{3}(x, y, t \mid u)$, of the surplus just before ruin, the deficit at ruin and the ruin time. Let $\delta \geq 0$ be the (constant) discount factor over one unit of time and define $f_{2}(x, y \mid u)=\int_{0}^{\infty} \mathrm{e}^{-\delta t} f_{3}(x, y, t \mid u) \mathrm{d} t$ to be a 'discounted' joint probability density function of $U\left(T^{-}\right)$and $|U(T)|$. Also define $g(y \mid u)=$ $\int_{0}^{\infty} f_{2}(x, y \mid u) \mathrm{d} x$. If $f_{1}(x \mid u)=\int_{0}^{\infty} f_{2}(x, y \mid u) \mathrm{d} y$ and $\bar{P}(x)=\int_{x}^{\infty} p(y) \mathrm{d} y=1-P(x)$, it follows from Gerber and Shiu (1998, Equation (2.40)) that

$$
f_{2}(x, y \mid u)=f_{1}(x \mid u) \frac{p(x+y)}{\bar{P}(x)}, \quad x, y, u \geq 0 .
$$


Let $w(x, y)$, for $x, y \geq 0$, be the nonnegative values of a penalty function. For $\delta>0$, define

$$
\phi(u)=\mathrm{E}\left[\mathrm{e}^{-\delta T} w\left(U\left(T^{-}\right),|U(T)|\right) \mathbf{1}(T<\infty) \mid U(0)=u\right], \quad u \geq 0 .
$$

The quantity $w\left(U\left(T^{-}\right),|U(T)|\right)$ can be interpreted as the penalty at the time of ruin for the surplus $U\left(T^{-}\right)$and the deficit $|U(T)|$. Then $\phi(u)$ is the expected discounted penalty if $\delta$ is viewed as the force of interest.

\section{Martingales and a generalized Lundberg fundamental equation}

Let $\tau_{k}=\sum_{j=1}^{k} W_{j}$, with $\tau_{0}=0$, denote the arrival time of the $k$ th claim and denote by $U_{k}=U\left(\tau_{k}\right)$ the surplus immediately after the $k$ th claim. Thus, $U_{0}=u$ while

$$
U_{k}=U\left(\tau_{k}\right)=u+c \tau_{k}-\sum_{j=1}^{k} X_{j}=u+\sum_{j=1}^{k}\left[c W_{j}-X_{j}\right] \quad \text { for } k \in \mathbb{N}^{+}
$$

We seek a function $v$ such that the process $V_{0}=\left\{\mathrm{e}^{-\delta \tau_{k}} v\left(U_{k}\right), k \in \mathbb{N}\right\}$ will form a martingale. Define $\mathcal{F}_{0}=\{\varnothing, \Omega\}$ and $\mathcal{F}_{k}$ to be the $\sigma$-algebra generated by $W_{1}, W_{2}, \ldots, W_{k}, X_{1}, X_{2}, \ldots, X_{k}$, representing the information of the surplus process immediately after the $k$ th claim, $k \in \mathbb{N}^{+}$. Then, by the definition of a discrete martingale,

$$
\mathrm{E}\left[\mathrm{e}^{-\delta \tau_{k+1}} v\left(U_{k+1}\right) \mid \mathcal{F}_{k}\right]=\mathrm{e}^{-\delta \tau_{k}} v\left(U_{k}\right), \quad k \in \mathbb{N} .
$$

Equation (2) is equivalent to

$$
\mathrm{E}\left[\mathrm{e}^{-\delta W_{k+1}} v\left(U_{k}+\left(c W_{k+1}-X_{k+1}\right)\right) \mid \mathcal{F}_{k}\right]=v\left(U_{k}\right), \quad k \in \mathbb{N} .
$$

In turn, this equation is equivalent to

$$
v(u)=\mathrm{E}\left[\mathrm{e}^{-\delta W} v(u+c W-X)\right]=\int_{0}^{\infty} \mathrm{e}^{-\delta t} k(t) \mathrm{E}[v(u+c t-X)] \mathrm{d} t .
$$

Equation (3) is a necessary and sufficient condition for the process $V_{0}$ to be a martingale.

As in Gerber and Shiu (1998), we choose $v(u)=\mathrm{e}^{s u}$ such that $\left\{\mathrm{e}^{-\delta \tau_{k}+s U_{k}}, k \in \mathbb{N}\right\}$ is a martingale. Then (3) simplifies to

$$
\gamma(s):=\frac{1}{\hat{k}(\delta-c s)}=\hat{p}(s), \quad s \in \mathbb{C},
$$

which is a generalization of Lundberg's fundamental equation.

Now we claim that $\left\{\mathrm{e}^{-\delta \tau_{k}} \phi\left(U_{k}\right), k \in \mathbb{N}\right\}$ is a martingale. To show this, let

$$
D=\mathrm{e}^{-\delta T} w\left(U\left(T^{-}\right),|U(T)|\right) \mathbf{1}(T<\infty)
$$

and define $M_{k}=\mathrm{E}\left[D \mid \mathcal{F}_{k}\right]$. It is easy to prove that $\left\{M_{k}, k \in \mathbb{N}\right\}$ is a martingale. Then

$$
\begin{aligned}
\phi(u) & =\mathrm{E}\left[M_{0}\right]=\mathrm{E}\left[M_{1}\right]=\mathrm{E}\left[\mathrm{E}\left[D \mid \mathcal{F}_{1}\right]\right]=\mathrm{E}\left[\mathrm{e}^{-\delta W_{1}} \phi\left(U_{1}\right)\right] \\
& =\mathrm{E}\left[\mathrm{e}^{-\delta W_{1}} \phi\left(u+c W_{1}-X_{1}\right)\right]=\int_{0}^{\infty} \mathrm{e}^{-\delta t} k(t) \mathrm{E}\left[\phi\left(u+c t-X_{1}\right)\right] \mathrm{d} t .
\end{aligned}
$$

This shows that $\left\{\mathrm{e}^{-\delta \tau_{k}} \phi\left(U_{k}\right), k \in \mathbb{N}\right\}$ is a martingale. 
Henceforth, we assume that $k$ belongs to the $K_{n}$ family of distributions, that is, the LT $\hat{k}(s)$ of the density $k$ is given by

$$
\hat{k}(s)=\frac{\lambda^{*}+s \beta(s)}{\prod_{i=1}^{n}\left(s+\lambda_{i}\right)},
$$

where $\lambda^{*}=\prod_{i=1}^{n} \lambda_{i}$, for $\lambda_{i}>0$ and $i=1,2, \ldots, n$, and $\beta(s)=\sum_{i=0}^{n-2} \beta_{i} s^{i}$ is a polynomial of degree $n-2$ or less. Then, the mean and variance of the interclaim time random variables are given by

$$
\mathrm{E}[W]=\sum_{i=1}^{n} \frac{1}{\lambda_{i}}-\frac{\beta(0)}{\lambda^{*}} \quad \text { and } \quad \operatorname{var}(W)=\sum_{i=1}^{n} \frac{1}{\lambda_{i}^{2}}+\frac{2 \beta^{\prime}(0) \lambda^{*}-\beta^{2}(0)}{\left(\lambda^{*}\right)^{2}},
$$

respectively. The class of $K_{n}$ distributions is widely used in applied probability applications (Cohen (1982), Tijms (1994)).

In this case, the generalized Lundberg equation in (4) simplifies to

$$
\gamma(s):=\frac{\prod_{i=1}^{n}\left(\lambda_{i}+\delta-c s\right)}{\lambda^{*}+(\delta-c s) \beta(\delta-c s)}=\hat{p}(s), \quad s \in \mathbb{C} .
$$

The following theorem shows that exactly $n$ of the roots of Lundberg's generalized equation (6) lie in the right-half complex plane. These play an important role here.

Theorem 1. For $\delta>0$ and $n \in \mathbb{N}^{+}$fixed, Lundberg's equation (6) has exactly $n$ roots, say $\rho_{1}(\delta), \rho_{2}(\delta), \ldots, \rho_{n}(\delta)$, that have a positive real part $\operatorname{Re}\left(\rho_{j}\right)>0$.

To prove this result, consider the half-circle in the complex plane given by $|z|=r$ (for $r>0$ fixed) and $\operatorname{Re}(z) \geq 0$. We have $|\gamma(s)|>1$ if $r$ is sufficiently large, while, for $s$ on the imaginary axis $(\operatorname{Re}(s)=0)$, we have $|\gamma(s)| \geq 1 /|\hat{k}(\delta-c s)|>1$. That is, on the contour boundary of the half-circle and the imaginary axis, $|\gamma(s)|>|\hat{p}(s)|$. We therefore conclude that, in the right-half plane, the number of roots of Lundberg's equation equals the number of roots of $\gamma(s)=0$. Since the latter has exactly $n$ positive roots, we deduce that (6) has exactly $n$ roots, say $\rho_{1}(\delta), \rho_{2}(\delta), \ldots, \rho_{n}(\delta)$, with positive real parts.

Remark 1. 1. Define $l(s):=\hat{p}(s)-\gamma(s)$. Since $l(0)<0$ and $\lim _{s \rightarrow-\infty} l(s)=\infty$, for $p$ sufficiently regular there is one negative root of $l(s)=0$, say $-R(\delta)$. We call $R(\delta)>0$ a generalized adjustment coefficient.

2. If $\delta \rightarrow 0^{+}$then $-R(\delta) \rightarrow-R(0)$ and $\rho_{j}(\delta) \rightarrow \rho_{j}(0)$, for $1 \leq j \leq n$, with $\rho_{n}(0)=0$, where $-R(0)$ and $\rho_{j}(0)$ are roots of the equation

$$
\gamma_{0}(s):=\frac{\prod_{i=1}^{n}\left(\lambda_{i}-c s\right)}{\lambda^{*}-c s \beta(-c s)}=\hat{p}(s), \quad s \in \mathbb{C} .
$$

3. For simplicity, write $-R$ and $\rho_{j}$ for $-R(\delta)$ and $\rho_{j}(\delta), 1 \leq j \leq n$, when $\delta>0$.

\section{The Laplace transform of $\phi(u)$}

In this section, we find the Laplace transform of the expected discounted penalty function $\phi(u)$. By (3),

$$
\phi(u)=\int_{0}^{\infty} \mathrm{e}^{-\delta t} k(t) \mathrm{E}[\phi(u+c t-X)] \mathrm{d} t .
$$


Setting $y=u+c t$ yields

$$
c \phi(u)=\int_{u}^{\infty} \exp \left[-\frac{\delta(y-u)}{c}\right] k\left(\frac{y-u}{c}\right) \mathrm{E}[\phi(y-X)] \mathrm{d} y .
$$

Making LTs gives

$$
\begin{aligned}
c \hat{\phi}(s) & =\int_{0}^{\infty} \mathrm{e}^{-s u} \int_{u}^{\infty} \exp \left[-\frac{\delta(y-u)}{c}\right] k\left(\frac{y-u}{c}\right) \mathrm{E}[\phi(y-X)] \mathrm{d} y \mathrm{~d} u \\
& =\int_{0}^{\infty} \mathrm{e}^{-\delta y / c} \mathrm{E}[\phi(y-X)] \int_{0}^{y} \exp \left[-\left(\frac{c s-\delta}{c}\right) u\right] k\left(\frac{y-u}{c}\right) \mathrm{d} u \mathrm{~d} y .
\end{aligned}
$$

First, if $\lambda_{1}, \lambda_{2}, \ldots, \lambda_{n}$ are distinct then, using partial fractions,

$$
\hat{k}(s)=\frac{\lambda^{*}+s \beta(s)}{\prod_{i=1}^{n}\left(s+\lambda_{i}\right)}=\sum_{i=1}^{n} \frac{a_{i}}{s+\lambda_{i}}, \quad s \in \mathbb{C},
$$

where $a_{i}=\left(\lambda^{*}-\lambda_{i} \beta\left(-\lambda_{i}\right)\right) / \prod_{j=1, j \neq i}^{n}\left(\lambda_{j}-\lambda_{i}\right)$. This gives $k(t)=\sum_{i=1}^{n} a_{i} \mathrm{e}^{-\lambda_{i} t} \mathbf{1}(t \geq 0)$. Then (7) becomes

$$
\begin{gathered}
c \hat{\phi}(s) \\
=\int_{0}^{\infty} \mathrm{e}^{-\delta y / c} \mathrm{E}[\phi(y-X)] \sum_{i=1}^{n} a_{i} \int_{0}^{y} \exp \left[-\left(\frac{c s-\delta}{c}\right) u\right] \exp \left[-\lambda_{i}\left(\frac{y-u}{c}\right)\right] \mathrm{d} u \mathrm{~d} y \\
=\sum_{i=1}^{n} a_{i} \int_{0}^{\infty} \exp \left[-\left(\frac{\delta+\lambda_{i}}{c}\right) y\right] \mathrm{E}[\phi(y-X)] \int_{0}^{y} \exp \left[-\left(\frac{c s-\delta-\lambda_{i}}{c}\right) u\right] \mathrm{d} u \mathrm{~d} y \\
=\sum_{i=1}^{n} \frac{c a_{i}}{c s-\delta-\lambda_{i}}\left\{\int_{0}^{\infty} \exp \left[-\left(\frac{\delta+\lambda_{i}}{c}\right) y\right] \mathrm{E}[\phi(y-X)] \mathrm{d} y\right. \\
\left.-\int_{0}^{\infty} \mathrm{e}^{-s y} \mathrm{E}[\phi(y-X)] \mathrm{d} y\right\} .
\end{gathered}
$$

Then, from (8),

$$
\hat{\phi}(s)=\sum_{i=1}^{n} \frac{a_{i} e_{i}}{c s-\delta-\lambda_{i}}+\hat{k}(\delta-c s) \int_{0}^{\infty} \mathrm{e}^{-s y} \mathrm{E}[\phi(y-X)] \mathrm{d} y,
$$

where

$$
e_{i}=\int_{0}^{\infty} \exp \left[-\left(\frac{\delta+\lambda_{i}}{c}\right) y\right] \mathrm{E}[\phi(y-X)] \mathrm{d} y,
$$

for $i=1,2, \ldots, n$. Since

$$
\mathrm{E}[\phi(y-X)]=\int_{0}^{y} \phi(y-x) p(x) \mathrm{d} x+\omega(y),
$$

where

$$
\omega(y)=\int_{y}^{\infty} w(y, x-y) p(x) \mathrm{d} x
$$


(9) reduces to

$$
\hat{\phi}(s)=\frac{\sum_{i=1}^{n} a_{i} e_{i} /\left(c s-\delta-\lambda_{i}\right)+\hat{k}(\delta-c s) \hat{\omega}(s)}{1-\hat{k}(\delta-c s) \hat{p}(s)},
$$

where $\hat{\omega}$ is the LT of $\omega$.

If, instead, some of the $\lambda_{i}$ in (5) are not distinct, then

$$
\hat{k}(s)=\frac{\prod_{i=1}^{k} \lambda_{i}^{n_{i}}+s \beta(s)}{\prod_{i=1}^{k}\left(s+\lambda_{i}\right)^{n_{i}}},
$$

where $\lambda_{1}, \lambda_{2}, \ldots, \lambda_{k}$ are distinct, $\lambda^{*}=\prod_{i=1}^{k} \lambda_{i}^{n_{i}}$, and $\sum_{i=1}^{k} n_{i}=n$. Then, using partial fractions,

$$
\hat{k}(s)=\frac{\lambda^{*}+s \beta(s)}{\prod_{i=1}^{k}\left(s+\lambda_{i}\right)^{n_{i}}}=\sum_{i=1}^{k} \sum_{j=1}^{n_{i}} \frac{a_{i, j}}{\left(s+\lambda_{i}\right)^{j}},
$$

where

$$
a_{i, j}=\left.\frac{1}{\left(n_{i}-j\right) !} \frac{\mathrm{d}^{n_{i}-j}}{\mathrm{~d} s^{n_{i}-j}} \prod_{m=1, m \neq i}^{k} \frac{\lambda^{*}+s \beta(s)}{\left(s+\lambda_{m}\right)^{n_{m}}}\right|_{s=-\lambda_{i}},
$$

and, hence,

$$
k(t)=\sum_{i=1}^{k} \sum_{j=1}^{n_{i}} a_{i, j} \frac{t^{j-1} \mathrm{e}^{-\lambda_{i} t}}{(j-1) !}, \quad t>0 .
$$

By a similar argument,

$$
\hat{\phi}(s)=\frac{-\sum_{i=1}^{k} \sum_{j=1}^{n_{i}} \sum_{m=0}^{j-1} a_{i, j} e_{i, m} /\left[c^{m} m !\left(\delta+\lambda_{i}-c s\right)^{j-m}\right]+\hat{k}(\delta-c s) \hat{\omega}(s)}{1-\hat{k}(\delta-c s) \hat{p}(s)},
$$

where

$$
e_{i, m}=\int_{0}^{\infty} y^{m} \exp \left[-\left(\frac{\delta+\lambda_{i}}{c}\right) y\right] \mathrm{E}[\phi(y-X)] \mathrm{d} y
$$

for $i=1,2, \ldots, k<n$.

The following theorem shows that, in both cases above, $\hat{\phi}(s)$ is given by the same expression.

Theorem 2. If the density function $k$ is a $K_{n}$ distribution, with $\hat{k}(s)$ being of the form in (5), then the LT of $\phi$ is given by

$$
\hat{\phi}(s)=\frac{\hat{\omega}(s)-q(s) /\left[\lambda^{*}+(\delta-c s) \beta(\delta-c s)\right]}{\gamma(s)-\hat{p}(s)},
$$

where $\gamma(s)$ is given in (6) and $q(s)$ is a polynomial of degree $n-1$, or less, determined by the following conditions:

$$
q\left(\rho_{j}\right)=\hat{\omega}\left(\rho_{j}\right)\left[\lambda^{*}+\left(\delta-c \rho_{j}\right) \beta\left(\delta-c \rho_{j}\right)\right], \quad j=1,2, \ldots, n .
$$

Furthermore, if $\rho_{1}, \rho_{2}, \ldots, \rho_{n}$ are distinct then

$$
q(s)=\sum_{j=1}^{n} \hat{\omega}\left(\rho_{j}\right)\left[\lambda^{*}+\left(\delta-c \rho_{j}\right) \beta\left(\delta-c \rho_{j}\right)\right] \prod_{k=1, k \neq j}^{n} \frac{s-\rho_{k}}{\rho_{j}-\rho_{k}} .
$$


Proof. If $\lambda_{1}, \lambda_{2}, \ldots, \lambda_{n}$ are distinct, then multiplying both the numerator and denominator of (10) by $\gamma(s)=1 / \hat{k}(\delta-c s)$ yields (12), with

$$
q(s)=\left[\prod_{i=1}^{n}\left(\delta+\lambda_{i}-c s\right)\right]\left[\sum_{i=1}^{n} \frac{a_{i} e_{i}}{\delta+\lambda_{i}-c s}\right]
$$

a polynomial of degree $n-1$ or less. Since $\hat{\phi}(s)$ is finite for all $s$ with $\operatorname{Re}(s)>0$, and we note that the $\rho_{j}$ (with $\left.\operatorname{Re}\left(\rho_{j}\right)>0, j=1,2, \ldots, n\right)$ are zeros of the denominator of $(12)$, then they must also be zeros of the numerator; that is to say, (13) holds. Furthermore, if $\rho_{1}, \rho_{2}, \ldots, \rho_{n}$ are distinct then, by the Lagrange interpolation formula, we obtain (14).

If

$$
\hat{k}(s)=\frac{\lambda^{*}+s \beta(s)}{\prod_{i=1}^{k}\left(s+\lambda_{i}\right)^{n_{i}}},
$$

with $\sum_{i=1}^{k} n_{i}=n$, then multiplying both the numerator and denominator of (11) by $\gamma(s)=$ $1 / \hat{k}(\delta-c s)$ gives (12), but this time with

$$
q(s)=\left[\prod_{i=1}^{k}\left(\delta+\lambda_{i}-c s\right)^{n_{i}}\right]\left[\sum_{i=1}^{k} \sum_{j=1}^{n_{i}} \sum_{m=0}^{j-1} \frac{a_{i, j} e_{i, m}}{c^{m} m !\left(\delta+\lambda_{i}-c s\right)^{j-m}}\right]
$$

a polynomial of degree $\leq n-1$, which can also be determined from (13) and obtained explicitly from (14) if $\rho_{1}, \ldots, \rho_{n}$ are distinct.

Remark 2. If

$$
\hat{k}(s)=\frac{\lambda^{*}}{\prod_{i=1}^{n}\left(s+\lambda_{i}\right)},
$$

that is, $p$ is generalized Erlang(n) distributed, then (12) simplifies to Gerber and Shiu (2005, Equation (7.3)). Moreover, for $n=1$ this formula can be found in the discussion, Dickson (1998a), of Gerber and Shiu (1998).

In the evaluation of the expected discounted penalty function, usually an integrodifferential equation satisfied by the expected discounted penalty function is first derived and then solved to produce a defective renewal equation; see Gerber and Shiu (1998), Dickson (1998b), Dickson and Hipp (1998), (2001), Cheng and Tang (2003), Li and Garrido (2004), and Gerber and Shiu (2003), (2005). Here it should be pointed out that, when $\beta(s) \not \equiv 0$, integrodifferential equations do not exist for the expected discounted penalty function $\phi(u)$ since, otherwise, there would exist a polynomial $h_{m}(s)$, of degree $m$, such that

$$
h_{m}(\mathscr{D}) \phi(u)=\int_{0}^{u} \phi(u-x) p(x) \mathrm{d} x+\omega(u), \quad u \geq 0,
$$

where $\mathscr{D}$ is a differentiation operator. Then, making LTs gives

$$
\hat{\phi}(s)=\frac{\hat{\omega}(s)-g_{m-1}(s)}{h_{m}(s)-\hat{p}(s)},
$$

with $g_{m-1}(s)$ a polynomial of degree $m-1$ or less. Theorem 2 shows that $h_{m}(s)=\gamma(s)$. When $\beta(s) \not \equiv 0, \gamma(s)$ is not a polynomial, which contradicts the assumption that $h_{m}(s)$ is a polynomial of degree $m$. 


\section{Analysis of the penalty function when $u=0$}

We now turn to the solution of our ruin-related problems when $u=0$. Henceforth, we assume that $\rho_{1}, \rho_{2}, \ldots, \rho_{n}$ in Theorem 1 are distinct. The analysis of the case in which $\rho_{1}, \rho_{2}, \ldots, \rho_{n}$ are not distinct is more tedious. First, by applying the initial value theorem,

$$
\begin{aligned}
\phi(0) & \\
= & \lim _{s \rightarrow \infty} s \hat{\phi}(s) \\
= & \lim _{s \rightarrow \infty} s \frac{\hat{\omega}(s)-q(s) /\left[\lambda^{*}+(\delta-c s) \beta(\delta-c s)\right]}{\gamma(s)-\hat{p}(s)} \\
= & \lim _{s \rightarrow \infty}\left[\hat{\omega}(s)-\frac{\sum_{j=1}^{n}\left\{\hat{\omega}\left(\rho_{j}\right)\left[\lambda^{*}+\left(\delta-c \rho_{j}\right) \beta\left(\delta-c \rho_{j}\right)\right] \prod_{k=1, k \neq j}^{n}\left(s-\rho_{k}\right) /\left(\rho_{j}-\rho_{k}\right)\right\}}{\lambda^{*}+(\delta-c s) \beta(\delta-c s)}\right] \\
& \times\left[\frac{\prod_{i=1}^{n}\left(\delta+\lambda_{i}-c s\right)}{s\left[\lambda^{*}+(\delta-c s) \beta(\delta-c s)\right]}-\frac{\hat{p}(s)}{s}\right]^{-1} \\
= & -\sum_{j=1}^{n}\left\{\hat{\omega}\left(\rho_{j}\right)\left[\lambda^{*}+\left(\delta-c \rho_{j}\right) \beta\left(\delta-c \rho_{j}\right)\right] \prod_{k=1, k \neq j}^{n} 1 /\left(\rho_{j}-\rho_{k}\right)\right\} \\
= & \sum_{j=1}^{n} \hat{\omega}\left(\rho_{j}\right) b_{j},
\end{aligned}
$$

where $b_{j}=\left[\lambda^{*}+\left(\delta-c \rho_{j}\right) \beta\left(\delta-c \rho_{j}\right)\right] /\left[c^{n} \prod_{k=1, k \neq j}^{n}\left(\rho_{k}-\rho_{j}\right)\right]$. When $\beta(s) \equiv 0$, this becomes Gerber and Shiu (2005, Equation (8.1)).

Since

$$
\omega(x)=\int_{x}^{\infty} w(x, y-x) p(y) \mathrm{d} y=\int_{0}^{\infty} w(x, y) p(x+y) \mathrm{d} y,
$$

its LT is $\hat{\omega}(s)=\int_{0}^{\infty} \int_{0}^{\infty} \mathrm{e}^{-s x} w(x, y) p(x+y) \mathrm{d} x \mathrm{~d} y$ and, so, $\phi(0)$ can be rewritten as

$$
\phi(0)=\sum_{j=1}^{n} b_{j} \int_{0}^{\infty} \int_{0}^{\infty} \mathrm{e}^{-\rho_{j} x} w(x, y) p(x+y) \mathrm{d} x \mathrm{~d} y .
$$

On the other hand,

$$
\begin{aligned}
\phi(0) & =\mathrm{E}\left[\mathrm{e}^{-\delta T} w\left(U\left(T^{-}\right),|U(T)|\right) \mathbf{1}(T<\infty) \mid U(0)=0\right] \\
& =\int_{0}^{\infty} \int_{0}^{\infty} w(x, y) f_{2}(x, y \mid 0) \mathrm{d} y \mathrm{~d} x,
\end{aligned}
$$

where $f_{2}$ is as given in (1). Comparing these two formulae for $\phi(0)$ yields

$$
f_{2}(x, y \mid 0)=\sum_{j=1}^{n} b_{j} \mathrm{e}^{-\rho_{j} x} p(x+y),
$$

whence

$$
f_{1}(x \mid 0)=\int_{0}^{\infty} f_{2}(x, y \mid 0) \mathrm{d} y=\sum_{j=1}^{n} b_{j} \mathrm{e}^{-\rho_{j} x} \bar{P}(x)
$$


and

$$
\begin{aligned}
g(y) & :=g(y \mid 0)=\int_{0}^{\infty} f_{2}(x, y \mid 0) \mathrm{d} x \\
& =\sum_{j=1}^{n} b_{j} \int_{0}^{\infty} \mathrm{e}^{-\rho_{j} x} p(x+y) \mathrm{d} x=\sum_{j=1}^{n} b_{j} T_{\rho_{j}} p(y),
\end{aligned}
$$

where $T_{r}$ is an operator defined by

$$
T_{r} p(x)=\int_{x}^{\infty} \mathrm{e}^{-r(y-x)} p(y) \mathrm{d} y=\int_{0}^{\infty} \mathrm{e}^{-r x} p(x+y) \mathrm{d} x .
$$

The function $g(y)$ is a defective density and plays a very important role in this paper. Its LT is given by

$$
\begin{aligned}
\hat{g}(s)= & \int_{0}^{\infty} \mathrm{e}^{-s y} g(y \mid 0) \mathrm{d} y=T_{s} g(0)=\sum_{j=1}^{n} b_{j} T_{s} T_{\rho_{j}} p(0)=\sum_{j=1}^{n} b_{j} \frac{\hat{p}\left(\rho_{j}\right)-\hat{p}(s)}{s-\rho_{j}} \\
= & \sum_{j=1}^{n} \frac{\prod_{i=1}^{n}\left(\delta+\lambda_{i}-c \rho_{j}\right)}{c^{n}\left(s-\rho_{j}\right) \prod_{k=1, k \neq j}^{n}\left(\rho_{k}-\rho_{j}\right)} \\
& -\hat{p}(s) \sum_{j=1}^{n} \frac{\lambda^{*}+\left(\delta-c \rho_{j}\right) \beta\left(\delta-c \rho_{j}\right)}{c^{n}\left(s-\rho_{j}\right) \prod_{k=1, k \neq j}^{n}\left(\rho_{k}-\rho_{j}\right)} \\
= & \sum_{j=1}^{n} \frac{\prod_{i=1}^{n}\left[\left(\delta+\lambda_{i}-c s\right)+c\left(s-\rho_{j}\right)\right]}{c^{n}\left(s-\rho_{j}\right) \prod_{k=1, k \neq j}^{n}\left(\rho_{k}-\rho_{j}\right)} \\
& -\hat{p}(s) \sum_{j=1}^{n} \frac{\lambda^{*}+\sum_{m=0}^{n-2} \beta_{m}\left[\delta-c s+c\left(s-\rho_{j}\right)\right]^{m+1}}{c^{n}\left(s-\rho_{j}\right) \prod_{k=1, k \neq j}^{n}\left(\rho_{k}-\rho_{j}\right)}
\end{aligned}
$$

where $\beta_{0}, \ldots, \beta_{n-2}$ are the coefficients of the polynomial $\beta(s)$. Since

$$
\prod_{i=1}^{n}\left[\left(\delta+\lambda_{i}-c s\right)+c\left(s-\rho_{j}\right)\right]=\sum_{l=0}^{n} \sigma_{l} c^{n-l}\left(s-\rho_{j}\right)^{n-l},
$$

where

$$
\begin{aligned}
\sigma_{0} & =1 \\
\sigma_{1} & =\sum_{i=1}^{n}\left(\delta+\lambda_{i}-c s\right), \\
\sigma_{2} & =\sum_{1 \leq i<j \leq n}\left(\delta+\lambda_{i}-c s\right)\left(\delta+\lambda_{j}-c s\right), \\
\vdots & \vdots \\
\sigma_{n} & =\prod_{i=1}^{n}\left(\delta+\lambda_{i}-c s\right),
\end{aligned}
$$


the first term simplifies to

$$
\begin{aligned}
\sum_{j=1}^{n} \frac{\prod_{i=1}^{n}\left[\left(\delta+\lambda_{i}-c s\right)+c\left(s-\rho_{j}\right)\right]}{c^{n}\left(s-\rho_{j}\right) \prod_{k=1, k \neq j}^{n}\left(\rho_{k}-\rho_{j}\right)} & =\sum_{l=0}^{n} \sum_{j=1}^{n} \frac{\sigma_{l} c^{n-l}\left(s-\rho_{j}\right)^{n-l}}{c^{n}\left(s-\rho_{j}\right) \prod_{k=1, k \neq j}^{n}\left(\rho_{k}-\rho_{j}\right)} \\
& =1-\frac{\prod_{i=1}^{n}\left(\delta+\lambda_{i}-c s\right)}{c^{n} \prod_{i=1}^{n}\left(\rho_{i}-s\right)} .
\end{aligned}
$$

Equation (17) follows from the following identities from interpolation theory (for $n \geq 2$ ):

$$
\sum_{j=1}^{n} \frac{\left(s-\rho_{j}\right)^{m}}{\prod_{k=1, k \neq j}^{n}\left(\rho_{k}-\rho_{j}\right)}= \begin{cases}1, & m=n-1, \\ 0, & m=0,1,2, \ldots, n-2, \\ -1 / \prod_{i=1}^{n}\left(\rho_{i}-s\right), & m=-1,\end{cases}
$$

which can be proved by divided differences (see Li and Garrido (2004)). Similarly,

$$
\begin{aligned}
\hat{p}(s) \sum_{j=1}^{n} \frac{\lambda^{*}+\sum_{m=0}^{n-2} \beta_{m}\left[\delta-c s+c\left(s-\rho_{j}\right)\right]^{m+1}}{c^{n}\left(s-\rho_{j}\right) \prod_{k=1, k \neq j}^{n}\left(\rho_{k}-\rho_{j}\right)} & =-\hat{p}(s) \frac{\lambda^{*}+\sum_{m=0}^{n-2} \beta_{m}(\delta-c s)^{m+1}}{c^{n} \prod_{i=1}^{n}\left(\rho_{i}-s\right)} \\
& =-\hat{p}(s) \frac{\lambda^{*}+(\delta-c s) \beta(\delta-c s)}{c^{n} \prod_{i=1}^{n}\left(\rho_{i}-s\right)} .
\end{aligned}
$$

Thus, the LT of $g$ reduces to

$$
\hat{g}(s)=1-\left[\frac{\prod_{i=1}^{n}\left(\delta+\lambda_{i}-c s\right)-\hat{p}(s)\left[\lambda^{*}+(\delta-c s) \beta(\delta-c s)\right]}{c^{n} \prod_{i=1}^{n}\left(\rho_{i}-s\right)}\right] .
$$

Setting $w(x, y)=1$ implies that

$$
\begin{aligned}
\phi(0) & =\mathrm{E}\left[\mathrm{e}^{-\delta T} \mathbf{1}(T<\infty) \mid U(0)=0\right]=\int_{0}^{\infty} g(y \mid 0) \mathrm{d} y=\lim _{s \rightarrow 0} \hat{g}(s) \\
& =1-\frac{\prod_{i=1}^{n}\left(\lambda_{i}+\delta\right)-\left[\lambda^{*}+\delta \beta(\delta)\right]}{c^{n} \rho_{1} \rho_{2} \cdots \rho_{n}} .
\end{aligned}
$$

Note that, since $\hat{k}(\delta)=\left[\lambda^{*}+\delta \beta(\delta)\right] / \prod_{i=1}^{n}\left(\lambda_{i}+\delta\right)<1$, we have $\phi(0)<1$.

Finally,

$$
\begin{aligned}
\Psi(0) & =\lim _{\delta \rightarrow 0} \mathrm{E}\left[\mathrm{e}^{-\delta T} \mathbf{1}(T<\infty) \mid U(0)=0\right] \\
& =\lim _{\delta \rightarrow 0}\left[1-\frac{\prod_{i=1}^{n}\left(\lambda_{i}+\delta\right)-\lambda^{*}}{c^{n} \rho_{1} \rho_{2} \cdots \rho_{n}}+\frac{\sum_{m=0}^{n-2} \beta_{m} \delta^{m+1}}{c^{n} \rho_{1} \rho_{2} \cdots \rho_{n}}\right] \\
& =1-\lim _{\delta \rightarrow 0} \frac{\left[\prod_{i=1}^{n}\left(\lambda_{i}+\delta\right)-\lambda^{*}\right] / \delta}{\rho^{*}(0) \rho_{n}^{\prime}(0)}+\frac{\beta_{0}}{\rho^{*}(0) \rho_{n}^{\prime}(0)} \\
& =1-\frac{\lambda^{*} \sum_{i=1}^{n} 1 / \lambda_{i}-\beta_{0}}{\rho^{*}(0) \rho_{n}^{\prime}(0)}=1-\frac{\lambda^{*}[c \mathrm{E}[W]-\mathrm{E}[X]]}{\rho^{*}(0)}<1,
\end{aligned}
$$

where $\rho^{*}(0)=\prod_{i=1}^{n-1} \rho_{i}(0)$ and a prime denotes differentiation. In the last step, we use the facts that $\mathrm{E}[W]=-\hat{k}^{\prime}(0)=\left[\lambda^{*} \sum_{i=1}^{n} 1 / \lambda_{i}-\beta_{0}\right] / \lambda^{*}$ and $\rho_{n}^{\prime}(0)=\mathrm{E}[W] /[c \mathrm{E}[W]-\mathrm{E}[X]]$. The latter follows from differentiating with respect to $\delta$ on both sides of $1 / \hat{k}\left(\delta-c \rho_{n}(\delta)\right)=\hat{p}\left(\rho_{n}(\delta)\right)$, letting $\delta \rightarrow 0$, and noting that $\lim _{\delta \rightarrow 0} \rho_{n}(\delta) \rightarrow 0$. 
Remark 3. 1 . When $\beta(s) \equiv 0$, i.e. $\beta_{m}=0$ for $m=0,1, \ldots, n-2,(20)$ simplifies to Gerber and Shiu (2005, Equation (11.5)).

2. If $c=1$ then (21) simplifies to Willmot (1999, Equation (3.10)).

\section{Defective renewal equation}

By arguments similar to those in Gerber and Shiu (1998), we condition on the first time that the surplus falls below the initial level $u$, i.e.

$$
\begin{aligned}
\phi(u)= & \int_{0}^{u} \int_{0}^{\infty} \int_{0}^{\infty} \mathrm{e}^{-\delta t} \phi(u-y) f_{3}(x, y, t \mid 0) \mathrm{d} t \mathrm{~d} x \mathrm{~d} y \\
& +\int_{u}^{\infty} \int_{0}^{\infty} \int_{0}^{\infty} \mathrm{e}^{-\delta t} w(x+u, y-u) f_{3}(x, y, t \mid 0) \mathrm{d} t \mathrm{~d} x \mathrm{~d} y \\
= & \int_{0}^{u} \int_{0}^{\infty} \phi(u-y) f_{2}(x, y \mid 0) \mathrm{d} x \mathrm{~d} y \\
& +\int_{u}^{\infty} \int_{0}^{\infty} w(x+u, y-u) f_{2}(x, y \mid 0) \mathrm{d} x \mathrm{~d} y \\
= & \int_{0}^{u} \phi(u-y) g(y) \mathrm{d} y+H(u), \quad u \geq 0,
\end{aligned}
$$

where

$$
\begin{aligned}
H(u) & =\int_{u}^{\infty} \int_{0}^{\infty} w(x+u, y-u) f_{2}(x, y \mid 0) \mathrm{d} x \mathrm{~d} y \\
& =\int_{0}^{\infty} \int_{u}^{\infty} w(s, t) f_{2}(s-u, t+u \mid 0) \mathrm{d} s \mathrm{~d} t \\
& =\sum_{j=1}^{n} b_{j} \int_{u}^{\infty} \mathrm{e}^{-\rho_{j}(s-u)} \int_{0}^{\infty} w(s, t) p(s+t) \mathrm{d} t \mathrm{~d} s=\sum_{j=1}^{n} b_{j} T_{\rho_{j}} \omega(u) .
\end{aligned}
$$

Since $\int_{0}^{\infty} g(y) \mathrm{d} y<1$, (22) is a defective renewal equation. In particular, if $\beta(s) \equiv 0$, it simplifies to Gerber and Shiu (2005, Equation (9.2)).

Setting $w(x, y)=1$, we then obtain $\omega(u)=\bar{P}(u)=T_{0} p(u)$ and

$$
H(u)=\sum_{j=1}^{n} b_{j} T_{\rho_{j}} T_{0} p(u)=T_{0} g(u)=\int_{u}^{\infty} g(y) \mathrm{d} y .
$$

Therefore, the LT of $T, \phi_{T}(u):=\mathrm{E}\left[\mathrm{e}^{-\delta T} \mathbf{1}(T<\infty) \mid U(0)=u\right]$, satisfies the following defective renewal equation:

$$
\phi_{T}(u)=\int_{0}^{u} \phi_{T}(u-y) g(y) \mathrm{d} y+\int_{u}^{\infty} g(y) \mathrm{d} y, \quad u \geq 0 .
$$

Furthermore, if $\delta=0$ then (23) gives

$$
\Psi(u)=\int_{0}^{u} \Psi(u-y) g_{0}(y) \mathrm{d} y+\int_{u}^{\infty} g_{0}(y) \mathrm{d} y, \quad u \geq 0,
$$


where $g_{0}(y)$ can be obtained by taking limits. Since $\lim _{\delta \rightarrow 0} \rho_{i}(\delta) \rightarrow \rho_{i}(0)$ and $\lim _{\delta \rightarrow 0} \rho_{n}(\delta)=$ $\rho_{n}(0)=0$, we have

$$
\begin{aligned}
g_{0}(y)= & \lim _{\delta \rightarrow 0} g(y)=\lim _{\delta \rightarrow 0} \sum_{j=1}^{n} b_{j} T_{\rho_{j}} p(y) \\
= & \sum_{j=1}^{n-1} \frac{\lambda^{*}-c \rho_{j}(0) \beta\left(-c \rho_{j}(0)\right)}{c^{n}\left[-\rho_{j}(0)\right] \prod_{k=1, k \neq j}^{n-1}\left[\rho_{k}(0)-\rho_{j}(0)\right]} T_{\rho_{j}(0)} p(y)+\frac{\lambda^{*} T_{0} p(y)}{c^{n} \rho^{*}(0)} \\
= & -\frac{\lambda^{*}}{c^{n}} \sum_{j=1}^{n-1} \frac{T_{\rho_{j}(0)} p(y)}{\rho_{j}(0) \prod_{k=1, k \neq j}^{n-1}\left[\rho_{k}(0)-\rho_{j}(0)\right]}+\frac{\lambda^{*} T_{0} p(y)}{c^{n} \rho^{*}(0)} \\
& +\frac{1}{c^{n-1}} \sum_{j=1}^{n-1} \frac{\beta\left(-c \rho_{j}\right) T_{\rho_{j}(0)} p(y)}{\prod_{k=1, k \neq j}^{n-1}\left[\rho_{k}(0)-\rho_{j}(0)\right]} \\
= & \frac{\lambda^{*}}{c^{n}} \sum_{j=1}^{n-1} \frac{\left[T_{0} p(y)-T_{\rho_{j}(0)} p(y)\right] / \rho_{j}(0)}{\prod_{k=1, k \neq j}^{n-1}\left[\rho_{k}(0)-\rho_{j}(0)\right]}+\frac{1}{c^{n-1}} \sum_{j=1}^{n-1} \frac{\beta\left(-c \rho_{j}\right) T_{\rho_{j}(0)} p(y)}{\prod_{k=1, k \neq j}^{n-1}\left[\rho_{k}(0)-\rho_{j}(0)\right]} \\
= & \frac{\lambda^{*}}{c^{n}} \sum_{j=1}^{n-1} \frac{T_{\rho_{j}(0)} \bar{P}(y)}{\prod_{k=1, k \neq j}^{n-1}\left[\rho_{k}(0)-\rho_{j}(0)\right]}+\frac{1}{c^{n-1}} \sum_{j=1}^{n-1} \frac{\beta\left(-c \rho_{j}\right) T_{\rho_{j}(0)} p(y)}{\prod_{k=1, k \neq j}^{n-1}\left[\rho_{k}(0)-\rho_{j}(0)\right]},
\end{aligned}
$$

where the second last step follows from (18). Note that $T_{\rho_{j}(0)} T_{0} p(y)=T_{\rho_{j}(0)} \bar{P}(y)$, while $\int_{0}^{\infty} g_{0}(y) \mathrm{d} y=\Psi(0)<1$ is given by $(21)$.

We note that the LT of $g_{0}(y)$, for $c=1$, is given by Willmot (1999, Equation (3.11)); however, the inversion of the transform is rather complicated except for some special choices of $\beta$.

Now we turn to two special subclasses of distribution for two different choices of $\beta$. (Alternatively, other subclasses may be considered.)

Case 1. $(\beta(s) \equiv 0$ (generalized Erlang $(n)$ distribution).) In the case that $\beta(s) \equiv 0$, the interclaim times distribution is the sum of $n$ exponential distributions with parameters $\lambda_{1}, \lambda_{2}, \ldots, \lambda_{n}$ and is called a generalized $\operatorname{Erlang}(n)$ distribution. In particular, if $\lambda_{i}=\lambda>0$ for all $i=1,2, \ldots, n$, then it is the $\operatorname{Erlang}(n)$ distribution. In general,

$$
\begin{aligned}
H(u) & =\frac{\lambda^{*}}{c^{n}} \sum_{j=1}^{n} \frac{T_{\rho_{j}} \omega(u)}{\prod_{k=1, k \neq j}^{n}\left(\rho_{k}-\rho_{j}\right)}, \\
g(y) & =\frac{\lambda^{*}}{c^{n}} \sum_{j=1}^{n} \frac{T_{\rho_{j}} p(y)}{\prod_{k=1, k \neq j}^{n}\left(\rho_{k}-\rho_{j}\right)}, \\
g_{0}(y) & =\frac{\lambda^{*}}{c^{n}} \sum_{j=1}^{n-1} \frac{T_{\rho_{j}(0)} \bar{P}(y)}{\prod_{k=1, k \neq j}^{n-1}\left[\rho_{k}(0)-\rho_{j}(0)\right]} .
\end{aligned}
$$

The above equations can be found in Gerber and Shiu (2005), and Li and Garrido (2004) for the case $\lambda_{i}=\lambda$. 
Case 2. $(\beta(s)=\beta$ (mixed exponential distribution).) In the case that $\beta(s)=\beta$, we have

$$
\begin{aligned}
H(u)= & \sum_{j=1}^{n} \frac{\lambda^{*}+\beta \delta-c \beta \rho_{j}}{c^{n} \prod_{k=1, k \neq j}^{n}\left(\rho_{k}-\rho_{j}\right)} T_{\rho_{j}} \omega(u), \\
g(y)= & \sum_{j=1}^{n} \frac{\lambda^{*}+\beta \delta-c \beta \rho_{j}}{c^{n} \prod_{k=1, k \neq j}^{n}\left(\rho_{k}-\rho_{j}\right)} T_{\rho_{j}} p(y), \\
g_{0}(y)= & \frac{\lambda^{*}}{c^{n}} \sum_{j=1}^{n-1} \frac{T_{\rho_{j}(0)} \bar{P}(y)}{\prod_{k=1, k \neq j}^{n-1}\left[\rho_{k}(0)-\rho_{j}(0)\right]} \\
& +\frac{\beta}{c^{n-1}} \sum_{j=1}^{n-1} \frac{T_{\rho_{j}(0)} p(y)}{\prod_{k=1, k \neq j}^{n-1}\left[\rho_{k}(0)-\rho_{j}(0)\right]} .
\end{aligned}
$$

In particular, if $n=2$ and the density function $k$ is the mixture of two exponential distributions, i.e. $k(x)=\left\{\theta \lambda_{1} \mathrm{e}^{-\lambda_{1} x}+(1-\theta) \lambda_{2} \mathrm{e}^{-\lambda_{2} x}\right\} \mathbf{1}(x>0)$, then $\beta=\theta \lambda_{1}+(1-\theta) \lambda_{2}$ and

$$
\begin{aligned}
g(y) & =\frac{\lambda_{1}+\lambda_{2}+\beta \delta-c \beta \rho_{2}}{c^{2}} T_{\rho_{2}} T_{\rho_{1}} p(y)+\frac{\beta}{c} T_{\rho_{1}} p(y), \\
g_{0}(y) & =\frac{\lambda_{1} \lambda_{2}}{c^{2}} T_{\rho_{1}(0)} \bar{P}(y)+\frac{\beta}{c} T_{\rho_{1}(0)} p(y) .
\end{aligned}
$$

\section{Discounted distributions of surplus before ruin and deficit at ruin}

In this section, we consider the discounted joint and marginal distributions of $U\left(T^{-}\right)$and $|U(T)|$ using the defective renewal equation (22).

Theorem 3. For $x, y, u \geq 0$, we have

$$
f_{2}(x, y \mid u)=\int_{0}^{u} f_{2}(x, y \mid u-z) g(z) \mathrm{d} z+\mathbf{1}(u<x) f_{2}(x-u, u+y \mid 0),
$$

where $f_{2}(x-u, y+u \mid 0)$ can be derived using (16).

Proof. If we set $w\left(x_{1}, x_{2}\right)=\mathbf{1}\left(x_{1}=x, y_{1}=y\right)$ then $\phi(u)$ in (22) simplifies to $f_{2}(x, y \mid u)$. This completes the proof.

Next, a closed form for the discounted marginal density $f_{1}(x \mid u)$ is obtained by inverting the discounted LT of $U\left(T^{-}\right)$. For notational convenience, let $\xi_{\delta}$ be such that

$$
\frac{1}{1+\xi_{\delta}}=\int_{0}^{\infty} g(y) \mathrm{d} y=\phi_{T}(0)
$$

and let $\xi_{0}$ be such that $1 /\left(1+\xi_{0}\right)=\int_{0}^{\infty} g_{0}(y) \mathrm{d} y=\Psi(0)$.

Theorem 4. The discounted marginal density can be written as

$$
f_{1}(x \mid u)= \begin{cases}\frac{1+\xi_{\delta}}{\xi_{\delta}} \sum_{j=1}^{n} b_{j} \mathrm{e}^{-\rho_{j} x} \bar{P}(x)\left[\mathrm{e}^{\rho_{j} x} \Psi_{j}(u-x)-\Psi_{j}(u)\right], & 0 \leq x<u, \\ \frac{1+\xi_{\delta}}{\xi_{\delta}} \sum_{j=1}^{n} b_{j} \mathrm{e}^{-\rho_{j} x} \bar{P}(x)\left[\mathrm{e}^{\rho_{j} u}-\Psi_{j}(u)\right], & x \geq u,\end{cases}
$$


where

$$
\Psi_{j}(u):=\phi_{T}(u)+\int_{0}^{u} \phi_{T}(u-t) \rho_{j} \mathrm{e}^{\rho_{j} t} \mathrm{~d} t .
$$

Proof. If $w(x, y)=\mathrm{e}^{-s x}$ then $\phi(u)=\mathrm{E}\left[\mathrm{e}^{-\delta T} \mathrm{e}^{-s U\left(T^{-}\right)} \mathbf{1}(T<\infty) \mid u(0)=u\right]$ is the discounted LT of $U\left(T^{-}\right)$at $s$. Then $w(x)=\mathrm{e}^{-s x} \bar{P}(x)$ and, hence, from (22) we have

$$
\phi(u)=\frac{1}{1+\xi_{\delta}} \int_{0}^{u} \phi(u-y)\left(1+\xi_{\delta}\right) g(y) \mathrm{d} y+\frac{1}{1+\xi_{\delta}}\left(1+\xi_{\delta}\right) H(u),
$$

with

$$
H(u)=\sum_{j=1}^{n} b_{j} \mathrm{e}^{\rho_{k} u} \int_{u}^{\infty} \mathrm{e}^{-\left(\rho_{k}+s\right) x} \bar{P}(x) \mathrm{d} x
$$

and $b_{1}, b_{2}, \ldots, b_{n}$ as defined in (15). Using Lin and Willmot (1999, Theorem 1.1), we have

$$
\phi(u)=\frac{1}{\xi_{\delta}} \int_{0}^{u}\left[1-\phi_{T}(u-x)\right] \mathrm{d} H(x)+\frac{H(0)}{\xi_{\delta}}\left[1-\phi_{T}(u)\right], \quad u \geq 0 .
$$

Substituting into this the expression for $H(u)$, we obtain

$$
\begin{gathered}
\phi(u)=\frac{1+\xi_{\delta}}{\xi_{\delta}}\left\{\sum_{j=1}^{n} b_{j} \int_{0}^{u}\left[1-\phi_{T}(u-x)\right] \rho_{j} \mathrm{e}^{\rho_{j} x} \int_{x}^{\infty} \mathrm{e}^{-\left(\rho_{j}+s\right) t} \bar{P}(t) \mathrm{d} t \mathrm{~d} x\right. \\
\quad-\sum_{j=1}^{n} b_{j} \int_{0}^{u}\left[1-\phi_{T}(u-x)\right] \mathrm{e}^{-s x} \bar{P}(x) \mathrm{d} x \\
\left.+\sum_{j=1}^{n} b_{j} \int_{0}^{\infty} \mathrm{e}^{-s x} \mathrm{e}^{-\rho_{j} x} \bar{P}(x) \mathrm{d} x\left[1-\phi_{T}(u)\right]\right\},
\end{gathered}
$$

and changing the order of integration yields

$$
\begin{aligned}
& \phi(u)=\frac{1+\xi_{\delta}}{\xi_{\delta}}\left\{\sum_{j=1}^{n} b_{j} \int_{0}^{u} \mathrm{e}^{-s x} \mathrm{e}^{-\rho_{j} x} \bar{P}(x) \int_{0}^{x}\left[1-\phi_{T}(u-t)\right] \rho_{j} \mathrm{e}^{\rho_{j} t} \mathrm{~d} t \mathrm{~d} x\right. \\
&+\sum_{j=1}^{n} b_{j} \int_{u}^{\infty} \mathrm{e}^{-s x} \mathrm{e}^{-\rho_{j} x} \bar{P}(x) \int_{0}^{u}\left[1-\phi_{T}(u-t)\right] \rho_{j} \mathrm{e}^{\rho_{j} t} \mathrm{~d} t \mathrm{~d} x \\
&-\sum_{j=1}^{n} b_{j} \int_{0}^{u}\left[1-\phi_{T}(u-x)\right] \mathrm{e}^{-s x} \bar{P}(x) \mathrm{d} x \\
&\left.+\sum_{j=1}^{n} b_{j} \int_{0}^{\infty} \mathrm{e}^{-s x} \mathrm{e}^{-\rho_{j} x} \bar{P}(x) \mathrm{d} x\left[1-\phi_{T}(u)\right]\right\} .
\end{aligned}
$$

As

$$
\phi(u)=\mathrm{E}\left[\mathrm{e}^{-\delta T} \mathrm{e}^{-s U\left(T^{-}\right)} \mathbf{1}(T<\infty) \mid u(0)=u\right]=\int_{0}^{\infty} \mathrm{e}^{-s x} f_{1}(x \mid u) \mathrm{d} x,
$$

by inverting (and recalling the definition of $\Psi_{k}(u)$ ), we recover the result. 
To compute $f_{2}(x, y \mid u)$ and $g(y \mid u)$, we use relation (1) to obtain

$$
f_{2}(x, y \mid u)= \begin{cases}\frac{1+\xi_{\delta}}{\xi_{\delta}} \sum_{j=1}^{n} b_{j} \mathrm{e}^{-\rho_{j} x} p(x+y)\left[\mathrm{e}^{\rho_{j} x} \Psi_{j}(u-x)-\Psi_{j}(u)\right], & 0 \leq x<u, \\ \frac{1+\xi_{\delta}}{\xi_{\delta}} \sum_{j=1}^{n} b_{j} \mathrm{e}^{-\rho_{j} x} p(x+y)\left[\mathrm{e}^{\rho_{j} u}-\Psi_{j}(u)\right], & x \geq u,\end{cases}
$$

and note that $g(y \mid u)=\int_{0}^{\infty} f_{2}(x, y \mid u) \mathrm{d} x$.

When $\delta \rightarrow 0, f_{2}(x, y \mid u), f_{1}(x \mid u)$, and $g(y \mid u)$ reduce to the joint and marginal densities of $U\left(T^{-}\right)$and $|U(T)|$.

\section{Explicit results for rational claim severity distributions}

The above theorems show that the discounted joint and marginal distributions of $U\left(T^{-}\right)$and $|U(T)|$ can be derived explicitly whenever the function $\phi_{T}(u)$ is known explicitly. One of these cases is when the LT $\hat{\phi}_{T}(s)$ is a rational function. Then, by locating its poles and using partial fractions, we can determine $\phi_{T}(u)$. It follows from (23) that $\hat{\phi}_{T}(s)$ is a rational function if and only if the $\operatorname{LT} \hat{g}(s)$ is also a rational function. Now, by (19), $\hat{g}(s)$ is rational if and only if $\hat{p}(s)$ is also. Furthermore, by Lin and Willmot (1999, Theorem 1.1), the solution $\phi(u)$ to the defective renewal equation (22) can be expressed explicitly in terms of $\phi_{T}(u)$. Therefore, $\phi_{T}(u)$ plays an extremely important role in evaluating the expected discounted penalty function $\phi(u)$.

Definition 1. A probability measure $\mu$ on $\mathbb{R}$ is said to belong to $\mathcal{R}_{f}$ if its Laplace transform is a rational function (i.e. the ratio of two polynomials). If $\mu$ is concentrated on $\mathbb{R}^{+}$then it is said to belong to $\mathcal{R}_{f}^{+}$. In either case, the distribution is said to be rational.

In this section, we assume that the claim size density function $p$ belongs to $\mathcal{R}_{f}^{+}$(as $p$ is usually defined on $\mathbb{R}^{+}$). That is, for $m \in \mathbb{N}^{+}$we have

$$
\hat{p}(s)=\frac{Q_{m-1}(s)}{Q_{m}(s)}, \quad \text { with } Q_{m}(0)=Q_{m-1}(0) \text { and } \operatorname{Re}(s) \in\left(h_{X}, \infty\right),
$$

where the abscissa of holomorphy $h_{X}$ of the claim size random variable $X$ is defined as

$$
h_{X}:=\inf \left\{s \in \mathbb{R}: \mathrm{E}\left[\mathrm{e}^{-s X}\right]<\infty\right\},
$$

$Q_{m}$ is a polynomial of degree $m$ with leading coefficient 1 , and $Q_{m-1}$ is a polynomial of degree $m-1$ or less. Furthermore, since $\hat{p}(s)$ is finite for all $s$ with $\operatorname{Re}(s)>0$, the equation $Q_{m}(s)=0$ has no roots with negative real parts.

The class $\mathcal{R}_{f}^{+}$is a wide class of distribution, including the $K_{n}$ family $\left(n \in \mathbb{N}^{+}\right.$) and distributions with damped sine and cosine functions as part of their densities. For example, the density function $\frac{17}{13} \mathrm{e}^{-x}[1-\sin (4 x)] \mathbf{1}(x \geq 0)$ has a rational LT given by

$$
\frac{\frac{17}{13}\left(s^{2}-2 s+13\right)}{(s+1)\left[(s+1)^{2}+16\right]}
$$

and belongs to $\mathcal{R}_{f}^{+}$. More complete discussions of rational distributions can be found in Cox (1955), Neuts (1981, Chapter 2), or Dufresne (2001). 
We now turn to deriving $\phi_{T}(u)$ by inverting its LT. Making LTs on both sides of the defective renewal equation (23) and using (19) yields

$$
\begin{aligned}
& \hat{\phi}_{T}(s) \\
& \quad=\frac{\phi_{T}(0)-\hat{g}(s)}{s[1-\hat{g}(s)]} \\
& \quad=\frac{\prod_{i=1}^{n}\left(\delta+\lambda_{i}-c s\right)-\hat{p}(s)\left[\lambda^{*}+(\delta-c s) \beta(\delta-c s)\right]-c^{n}\left[1-\phi_{T}(0)\right] \prod_{i=1}^{n}\left(\rho_{i}-s\right)}{s\left[\prod_{i=1}^{n}\left(\delta+\lambda_{i}-c s\right)-\hat{p}(s)\right]\left[\lambda^{*}+(\delta-c s) \beta(\delta-c s)\right]} .
\end{aligned}
$$

When $p$ is a rational distribution, as in $(24), \hat{\phi}_{T}(s)$ admits a rational expression and we have the following results.

Theorem 5. If the Laplace transform $\hat{p}(s)$ of the claim size density is defined as in (24), then

$$
\hat{\phi}_{T}(s)=\frac{P_{m-1}(s)}{\left(s+R_{1}\right)\left(s+R_{2}\right) \cdots\left(s+R_{m}\right)}, \quad s \in \mathbb{C} .
$$

Furthermore, if $R_{1}, R_{2}, \ldots, R_{m}$ are distinct then

$$
\hat{\phi}_{T}(s)=\sum_{i=1}^{m} \frac{r_{i}}{s+R_{i}} \quad \text { and } \quad \phi_{T}(u)=\sum_{i=1}^{m} r_{i} \mathrm{e}^{-R_{i} u},
$$

where $P_{m-1}(s)=\left[\prod_{i=1}^{m}\left(s+R_{1}\right)-\phi_{T}(0) Q_{m}(s)\right] / s$ is a polynomial of degree $m-1$ or less, $r_{i}=\left(Q_{m}\left(-R_{i}\right) / Q_{m}(0)\right) \prod_{j=1, j \neq i}^{m} R_{j} /\left(R_{j}-R_{i}\right)$, and the $-R_{i}$, with $\operatorname{Re}\left(R_{i}\right)>0$ for $i=1,2, \ldots, m$, are those roots of the equation

$$
Q_{n, m}(s):=Q_{m}(s) \prod_{i=1}^{n}\left(\delta+\lambda_{i}-c s\right)-Q_{m-1}(s)\left[\lambda^{*}+(\delta-c s) \beta(\delta-c s)\right]=0
$$

that have negative real parts.

Proof. Substituting $\hat{p}(s)$ in (24) into (25), and multiplying both the numerator and denominator by $Q_{m}(s)$, gives

$$
\hat{\phi}_{T}(s)=\frac{Q_{n, m}(s)-c^{n}\left[1-\phi_{T}(0)\right] Q_{m}(s) \prod_{i=1}^{n}\left(\rho_{i}-s\right)}{s Q_{n, m}(s)}, \quad s \in \mathbb{C},
$$

where $Q_{n, m}(s)$ is, as above, a polynomial of degree $n+m$ with leading coefficient $(-c)^{n}$. In this case, the generalized Lundberg equation

$$
\frac{\prod_{i=1}^{n}\left(\delta+\lambda_{i}-c s\right)}{\lambda^{*}+(\delta-c s) \beta(\delta-c s)}=\frac{Q_{m-1}(s)}{Q_{m}(s)}
$$

is equivalent to $Q_{n, m}(s)=0$, for $\operatorname{Re}(s)>h_{X}$. It has $n$ roots, say $\rho_{1}, \rho_{2}, \ldots, \rho_{n}$, with positive real parts, and one root, say $-R$, with a negative real part, where $h_{X}<-R<0$. On the other hand the equation $Q_{n, m}(s)=0$ has $n+m$ roots, namely $\rho_{1}, \rho_{2}, \ldots, \rho_{n}$, with $\operatorname{Re}\left(\rho_{i}\right)>0$, and $-R_{i}$, with $\operatorname{Re}\left(R_{i}\right)>0$ for $i=1,2, \ldots, m$; of the latter, $R=\min \left\{\operatorname{Re}\left(R_{i}\right), i=1,2, \ldots, m\right\}$. We can express $Q_{n, m}(s)$ as

$$
Q_{n, m}(s)=c^{n} \prod_{i=1}^{m}\left(s+R_{i}\right) \prod_{j=1}^{n}\left(\rho_{j}-s\right)
$$


Substituting this into the expression for $\hat{\phi}_{T}(s)$ in (27), and simplifying, yields

$$
\hat{\phi}_{T}(s)=\frac{\prod_{i=1}^{m}\left(s+R_{i}\right)-\left[1-\phi_{T}(0)\right] Q_{m}(s)}{s \prod_{i=1}^{m}\left(s+R_{i}\right)}, \quad s \in \mathbb{C} .
$$

The numerator above must vanish if $s=0$, i.e. $1-\phi_{T}(0)=R_{1} R_{2} \cdots R_{m} / Q_{m}(0)$, since this point is a removable singularity. Therefore,

$$
P_{m-1}(s):=\frac{\prod_{i=1}^{m}\left(s+R_{1}\right)-\left[1-\phi_{T}(0)\right] Q_{m}(s)}{s}
$$

is a polynomial of degree $m-1$ or less.

When $R_{1}, R_{2}, \ldots, R_{m}$ are distinct, using partial fractions gives

$$
\hat{\phi}_{T}(s)=\frac{P_{m-1}(s)}{\prod_{i=1}^{m}\left(s+R_{i}\right)}=\sum_{i=1}^{m} \frac{r_{i}}{s+R_{i}},
$$

where

$$
r_{i}=\frac{P_{m-1}\left(-R_{i}\right)}{\prod_{j=1, j \neq i}^{m}\left(R_{j}-R_{i}\right)}=\frac{Q_{m}\left(-R_{i}\right)}{Q_{m}(0)} \prod_{j=1, j \neq i}^{m} \frac{R_{j}}{R_{j}-R_{i}} .
$$

Inverting this $\mathrm{LT}$ gives $\phi_{T}(u)=\sum_{i=1}^{m} r_{i} \mathrm{e}^{-R_{i} u}$, which proves (26).

Remark 4. 1. The fact that $P_{m-1}(s)$ must vanish at $s=0$ shows that

$$
1-\phi_{T}(0)=\frac{R_{1} R_{2} \cdots R_{m}}{Q_{m}(0)} .
$$

Therefore, $\phi_{T}(0)=1-R_{1} R_{2} \cdots R_{m} / Q_{m}(0)$ can be expressed in terms of the $R_{i}$. Of course, (20) still holds for $\phi_{T}(0)$.

2. If $\hat{p}(s)$ is as defined in (24), $\hat{g}(s)$ simplifies to

$$
\hat{g}(s)=\frac{Q_{m}(s)-\prod_{i=1}^{n}\left(s+R_{i}\right)}{Q_{m}(s)} .
$$

Note that (using partial fractions) $g(y)$ is of the same form as the claim density $p$.

3. We can also obtain $\phi_{T}(u)$ using the theorem of residues:

$$
\phi_{T}(u)=\sum_{i=1}^{m}\left\{\text { residue of } \mathrm{e}^{s u} \hat{\phi}_{T}(s) \text { at singularity }-R_{i}\right\} .
$$

Example 1. Assume that the interclaim times distribution $k$ belongs to the $K_{n}$ family given in (5), while the claim amounts are exponentially distributed, that is, $p(x)=a \mathrm{e}^{-a x} \mathbf{1}(x \geq 0)$, with $\hat{p}(s)=a /(s+a)$ and $a>0$. Then $\rho_{1}, \rho_{2}, \ldots, \rho_{n}$ and $-R<0$ are the $n+1$ roots of the equation

$$
Q_{n, 1}(s):=(s+a) \prod_{i=1}^{n}\left(\delta+\lambda_{i}-c s\right)-a\left[\lambda^{*}+(\delta-c s) \beta(\delta-c s)\right]=0 .
$$


Hence,

$$
\phi_{T}(u)=\int_{0}^{u} \phi_{T}(u-y) g(y) \mathrm{d} y+\int_{u}^{\infty} g(y) \mathrm{d} y, \quad u \geq 0,
$$

with $\phi_{T}(0)=1-R / a$, and (28) gives

$$
\hat{g}(s)=\frac{a-R}{s+a} \quad \text { and } \quad g(y)=(a-R) \mathrm{e}^{-a y} \mathbf{1}(y \geq 0) .
$$

Finally, Theorem 5 gives $\hat{\phi}_{T}(s)=\phi_{T}(0) /(s+R)$ and, therefore, the LT of $T$ is given by

$$
\phi_{T}(u)=\mathrm{E}\left[\mathrm{e}^{-\delta T} \mathbf{1}(T<\infty) \mid U(0)=u\right]=\phi_{T}(0) \mathrm{e}^{-R u}=\frac{a-R}{a} \mathrm{e}^{-R u},
$$

while the ultimate ruin probability is

$$
\Psi(u)=\mathrm{P}\{T<\infty \mid U(0)=u\}=\lim _{\delta \rightarrow 0} \phi_{T}(u)=\frac{a-R(0)}{a} \mathrm{e}^{-R(0) u},
$$

where $R(0)$ is the negative root of the generalized Lundberg equation with $\delta=0$. To compute the discounted joint and marginal distributions of $U\left(T^{-}\right)$and $|U(T)|$, we need to find $\Psi_{j}(u)$ in Theorem 4 for $j=1,2, \ldots, n$ and $u \geq 0$. These can be written as

$$
\Psi_{j}(u)=\phi_{T}(u)+\int_{0}^{u} \phi_{T}(u-t) \rho_{j} \mathrm{e}^{\rho_{j} t} \mathrm{~d} t=\frac{a-R}{a} \frac{1}{R+\rho_{j}}\left[R \mathrm{e}^{-R u}+\rho_{j} \mathrm{e}^{\rho_{j} u}\right] .
$$

Therefore, by Theorem 4, the discounted marginal distribution of $U\left(T^{-}\right)$is

$$
f_{1}(x \mid u)= \begin{cases}(a-R) \mathrm{e}^{-(R u+a x)} \sum_{j=1}^{n} \frac{b_{j}\left[\mathrm{e}^{R x}-\mathrm{e}^{-\rho_{j} x}\right]}{R+\rho_{j}}, & 0 \leq x \leq u, \\ \mathrm{e}^{-a x} \sum_{j=1}^{n}\left[\frac{b_{j}\left(a+\rho_{j}\right)}{R+\rho_{j}} \mathrm{e}^{-\rho_{j}(x-u)}-\frac{b_{j}(a-R)}{R+\rho_{j}} \mathrm{e}^{-\left(R u+\rho_{j} x\right)}\right], & x>u,\end{cases}
$$

where here

$$
b_{j}=\frac{\lambda^{*}+\left(\delta-c \rho_{j}\right) \beta\left(\delta-c \rho_{j}\right)}{c^{n} \prod_{k=1, k \neq j}^{n}\left(\rho_{k}-\rho_{j}\right)} .
$$

Finally, the relation $f_{2}(x, y \mid u)=f_{1}(x \mid u) p(x+y) / \bar{P}(x)$ yields

$$
f_{2}(x, y \mid u)=a \mathrm{e}^{-a y} f_{1}(x \mid u)
$$

and, hence,

$$
g(y \mid u)=\int_{0}^{\infty} f_{2}(x, y \mid u) \mathrm{d} x=a \mathrm{e}^{-a y} \phi_{T}(u)=(a-R) \mathrm{e}^{-(R u+a y)} .
$$

Example 2. Assume that the interclaim times are distributed as a mixture of two exponentials, with density $k(x)=\left[\theta \lambda_{1} \mathrm{e}^{-\lambda_{1} x}+(1-\theta) \lambda_{2} \mathrm{e}^{-\lambda_{2} x}\right] \mathbf{1}(x \geq 0)$. The LT of the density is

$$
\hat{k}(s)=\frac{\lambda_{1} \lambda_{2}+\left[\theta \lambda_{1}+(1-\theta) \lambda_{2}\right] s}{\left(s+\lambda_{1}\right)\left(s+\lambda_{2}\right)} .
$$


Here, the claim size density has a damped sine term, i.e.

$$
p(x)=\frac{a\left(a^{2}+b^{2}\right)}{a^{2}+b^{2}-a b} \mathrm{e}^{-a x}[1-\sin (b x)] \mathbf{1}(x \geq 0),
$$

with

$$
\hat{p}(s)=\frac{Q_{2}(s)}{Q_{3}(s)}=\frac{a\left(a^{2}+b^{2}\right)}{a^{2}+b^{2}-a b} \frac{s^{2}+(2 a-b) s+b^{2}+a^{2}-a b}{(s+a)\left[(s+a)^{2}+b^{2}\right]},
$$

where $a>0$ and $b>0$. Now,

$$
\begin{aligned}
0= & Q_{2,3}(s) \\
= & \left(a^{2}+b^{2}-a b\right)(s+a)\left[(s+a)^{2}+b^{2}\right]\left(\delta+\lambda_{1}-c s\right)\left(\delta+\lambda_{2}-c s\right) \\
& -a\left(a^{2}+b^{2}\right)\left[s^{2}+(2 a-b) s+b^{2}+a^{2}-a b\right]\left[\lambda_{1} \lambda_{2}+\left(\theta \lambda_{1}+(1-\theta) \lambda_{2}\right)(\delta-c s)\right]
\end{aligned}
$$

has two roots with positive real parts, say $\rho_{1}$ and $\rho_{2}$, and three roots with negative real parts (at least one real), say $-R_{1},-R_{2}$, and $-R_{3}\left(\operatorname{Re}\left(R_{i}\right)>0\right)$. Here,

$$
\phi_{T}(u)=\int_{0}^{u} \phi_{T}(u-y) g(y) \mathrm{d} y+\int_{u}^{\infty} g(y) \mathrm{d} y,
$$

where

$$
\phi_{T}(0)=1-\frac{R_{1} R_{2} R_{3}}{Q_{3}(0)}=1-\frac{R_{1} R_{2} R_{3}}{a\left(a^{2}+b^{2}\right)}
$$

Hence, (28) gives

$\hat{g}(s)$

$$
=\frac{\left(3 a-\sum_{i=1}^{3} R_{i}\right) s^{2}+\left(3 a^{2}+b^{2}-R_{1} R_{2}-R_{1} R_{3}-R_{2} R_{3}\right) s+a\left(a^{2}+b^{2}\right)-R_{1} R_{2} R_{3}}{(s+a)\left[(s+a)^{2}+b^{2}\right]},
$$

which has inverse

$$
g(y)=\mathrm{e}^{-a y}\left[\eta-\eta_{1} \cos (b y)-\eta_{2} \sin (b y)\right],
$$

where

$$
\begin{aligned}
b^{2} \eta & =a^{3}-a^{2}\left(R_{1}+R_{2}+R_{3}\right)+a\left(R_{1} R_{2}+R_{1} R_{3}+R_{2} R_{3}\right)-R_{1} R_{2} R_{3}, \\
b^{2} \eta_{1} & =a^{3}-3 a b^{2}+\left(b^{2}-a^{2}\right)\left(R_{1}+R_{2}+R_{3}\right)+a\left(R_{1} R_{2}+R_{1} R_{3}+R_{2} R_{3}\right)-R_{1} R_{2} R_{3}, \\
b^{2} \eta_{2} & =3 a^{2} b-b^{3}-2 a b\left(R_{1}+R_{2}+R_{3}\right)+b\left(R_{1} R_{2}+R_{1} R_{3}+R_{2} R_{3}\right) .
\end{aligned}
$$

Note that $g(y)$ is of the same form as the claim size density function. Here, Theorem 5 gives

$$
\phi_{T}(u)=\phi_{T}(0)\left[z_{1} \mathrm{e}^{-R_{1} u}+z_{2} \mathrm{e}^{-R_{2} u}+z_{3} \mathrm{e}^{-R_{3} u}\right], \quad u \geq 0,
$$

with

$$
\begin{aligned}
& z_{1}=\frac{\left(a-R_{1}\right)\left[\left(a-R_{1}\right)^{2}+b^{2}\right]}{a\left(a^{2}+b^{2}\right)-R_{1} R_{2} R_{3}} \frac{R_{2} R_{3}}{\left(R_{2}-R_{1}\right)\left(R_{3}-R_{1}\right)}, \\
& z_{2}=\frac{\left(a-R_{2}\right)\left[\left(a-R_{2}\right)^{2}+b^{2}\right]}{a\left(a^{2}+b^{2}\right)-R_{1} R_{2} R_{3}} \frac{R_{1} R_{3}}{\left(R_{1}-R_{2}\right)\left(R_{3}-R_{2}\right)}, \\
& z_{3}=1-z_{1}-z_{2} .
\end{aligned}
$$


Finally, in this example, Theorem 4 yields

$$
\begin{aligned}
\Psi_{j}(u) & =\phi_{T}(u)+\int_{0}^{u} \phi_{T}(u-y) \rho_{j} \mathrm{e}^{\rho_{j} y} \mathrm{~d} y \\
& =\phi_{T}(0)\left[\sum_{i=1}^{3} \pi_{j, i} \mathrm{e}^{-R_{i} u}+\left(1-\pi_{j, 1}-\pi_{j, 2}-\pi_{j, 3}\right) \mathrm{e}^{\rho_{j} u}\right],
\end{aligned}
$$

where $\pi_{j, i}=z_{i} R_{i} /\left(R_{i}+\rho_{j}\right), i=1,2,3, j=1,2$, which gives the discounted marginal distribution of $U\left(T^{-}\right)$as

$$
f_{1}(x \mid u)= \begin{cases}\frac{\phi_{T}(0)}{1-\phi_{T}(0)} \bar{P}(x) \sum_{j=1}^{2} b_{j} \sum_{i=1}^{3} \pi_{j, i} \mathrm{e}^{-R_{i} u}\left[\mathrm{e}^{R_{i} x}-\mathrm{e}^{-\rho_{j} x}\right], & 0 \leq x<u, \\ \frac{\phi_{T}(0)}{1-\phi_{T}(0)} \bar{P}(x) \sum_{j=1}^{2} b_{j} \mathrm{e}^{-\rho_{j} x} \sum_{i=1}^{3} \pi_{j, i}\left[\mathrm{e}^{\rho_{j} u}-\mathrm{e}^{-R_{i} u}\right], & x \geq u,\end{cases}
$$

where here

$$
b_{1}=\frac{\lambda_{1} \lambda_{2}+\left(\delta-c \rho_{1}\right)\left[\theta \lambda_{1}+(1-\theta) \lambda_{2}\right]}{c^{2}\left(\rho_{2}-\rho_{1}\right)} \quad \text { and } \quad b_{2}=\frac{\lambda_{1} \lambda_{2}+\left(\delta-c \rho_{2}\right)\left[\theta \lambda_{1}+(1-\theta) \lambda_{2}\right]}{c^{2}\left(\rho_{1}-\rho_{2}\right)} \text {. }
$$

\section{Concluding remarks}

We have shown how the evaluation of the Gerber-Shiu expected discounted penalty function for the classical risk model can be extended to a class of renewal risk processes with $K_{n}$-distributed interclaim times $\left(n \in \mathbb{N}^{+}\right)$. This leads to a defective renewal equation for the penalty function with a general claim severity distribution. Moreover, in cases in which the claim severities have a rational distribution, we have obtained explicit results using partial fractions.

The defective renewal equations obtained here can be used to solve other problems related to ruin: explicit expressions or bounds and asymptotic formulae for ruin probabilities, and joint and marginal distributions of the time to ruin, surplus before ruin, and deficit at ruin, as well as their moments.

These results can be extended to the case in which the interclaim times distribution belongs to the class $\mathcal{R}_{f}^{+}$, which is wider than the $K_{n}$ family.

\section{Acknowledgements}

The authors are thankful for the constructive comments of an anonymous referee. These helped improve the presentation of the results above. In this research, the first author was funded by a Society of Actuaries PhD grant and the second author by the Natural Sciences and Engineering Research Council of Canada (NSERC) operating grant OGP0036860.

\section{References}

Andersen, S. E. (1957). On the collective theory of risk in case of contagion between claims. Bull. Inst. Math. Appl. 12, 275-279.

Cheng, Y. And TANG, Q. (2003). Moments of the surplus before ruin and the deficit at ruin in the Erlang(2) risk process. N. Amer. Actuarial J. 7, 1-12.

CoHen, J. W. (1982). The Single Server Queue, 2nd edn. North-Holland, Amsterdam. 
Cox, D. R. (1955). A use of complex probabilities in the theory of stochastic processes. Proc. Camb. Philos. Soc. 51, 313-319.

Dickson, D. C. M. (1998a). Discussion on 'On the time value of ruin', by Gerber, H. U. and Shiu, E. S. W. N. Amer. Actuarial J. 2, 74.

Dickson, D. C. M. (1998b). On a class of renewal risk processes. N. Amer. Actuarial J. 2, 60-68.

Dickson, D. C. M. AND HipP, C. (1998). Ruin probabilities for Erlang(2) risk process. Insurance Math. Econom. 22, 251-262.

Dickson, D. C. M. AND Hipp, C. (2001). On the time to ruin for Erlang(2) risk process. Insurance Math. Econom. 29, 333-344.

Dufresne, D. (2001). On a general class of risk models. Austral. Actuarial J. 7, 755-791.

Gerber, H. U. AND ShiU, E. S. W. (1998). On the time value of ruin. N. Amer. Actuarial J. 2, 48-78.

Gerber, H. U. And Shiu, E. S. W. (2003). Discussion on 'Moments of the surplus before ruin and the deficit at ruin in the Erlang(2) risk process', by Y. Cheng and Q. Tang. N. Amer. Actuarial J. 7, 117-119.

Gerber, H. U. And Shiu, E. S. W. (2005). The time value of ruin in a Sparre Andersen model. N. Amer. Actuarial J. 9, 49-69.

LI, S. (2003). Discussion on 'Moments of the surplus before ruin and the deficit at ruin in the Erlang(2) risk process', by Y. Cheng and Q. Tang. N. Amer. Actuarial J. 7, 119-122.

LI, S. AND GARRIDO, J. (2004). 'On ruin for Erlang(n) risk process.' Insurance Math. Econom. 34, 391-408.

LIN, X. S. (2003). Discussion on 'Moments of the surplus before ruin and the deficit at ruin in the Erlang(2) risk process', by Y. Cheng and Q. Tang. N. Amer. Actuarial J. 7, 122-124.

Lin, X. S. AND WiLlmot, G. E. (1999). Analysis of a defective renewal equation arising in ruin theory. Insurance Math. Econom. 25, 63-84.

Neuts, M. F. (1981). Matrix-Geometric Solutions in Stochastic Models. Johns Hopkins University Press, Baltimore, MD.

Tusms, H. C. (1994). Stochastic Models. An Algorithmic Approach. John Wiley, Chichester.

Willmot, G. E. (1999). A Laplace transform representation in a class of renewal queueing and risk process. J. Appl. Prob. 36, 570-584. 